\title{
ON THE PRINCIPLE OF CONDITIONAL GHOST CORRECTION AND ITS REALIZATION IN EXISTING CORRECTION CONCEPTS
}

\section{S. MATTHIES}

Central Institute for Nuclear Research Rossendorf

Academy of Sciences of the GDR

8051 Dresden, DDR, PSF 19

\section{INTRODUCTION}

As it is well known the ("reduced") pole figures $\widetilde{P}_{\mathbf{h}}(\mathbf{y})$ derived by diffraction experiments contain less information than it is necessary for an unambiguos reproduction of the asked ODF $f(g)$. There are some reasons for such a loss of information ${ }^{1,2}$. They are connected with the fact that the properties of crystal symmetry of the pole figure data will be described by a point group of higher order than the corresponding rotation group for the ODF.

All formal solutions of the central problem of texture analysis

$$
\widetilde{P}_{\mathbf{h}_{i}}(\mathbf{y})->f(g), \text { with } \widetilde{P}_{\mathbf{h}_{i}}(\mathbf{y})=\widehat{\widetilde{P}}\left(\mathbf{h}_{i}, \mathbf{y}\right) f(g)=\widehat{\widetilde{P}}\left(\mathbf{h}_{i}, \mathbf{y}\right) \tilde{f}(g),
$$

can be represented by

$$
f^{M}(g)=\widetilde{f}(g)+\widetilde{f}^{M}(g)
$$

with $\widetilde{f}^{M}(g)$ belonging to the kernel $\widetilde{F}(g)$ of the operator $\widehat{\widetilde{P}}: \widehat{\widetilde{P}}\left(\mathbf{h}_{i}, \mathbf{y}\right) \widetilde{F}(g) \equiv 0$. The difficulties with the ambiguity of solutions would be artificial ones if the reduced ODF $\mathfrak{f}(g)$ were sufficient in order to characterize the orientation distribution of the crystallites in a sample. However, as a rule $\widetilde{f}(g)$ is not a positive function and contains ghost phenomena, e.g. maxima of intensity not existing in the true ODF.

The term ghost correction means the problem of reconstruction of the lost part $\widetilde{f}(g)$ of the true ODF resting upon reduced data $(\widetilde{P}$ or $\widetilde{f})$ only. Because of the kernel $\widetilde{F}$ this problem is mathematically senseless: $\widetilde{f}(g)$ is only one of the infinite number of possible $F^{M}(g)$. However, there are some physical arguments, expierence about the structure of real orientation distributions and at the end additional (artificial, subjective) conditions that can narrow the variation width of possible solutions in a maximum way.

The aim of the paper is to demonstrate and to discuss new activities and knowledge in this direction as a direct continuation of our contributions to this nontrivial and complex matter on ICOTOM $6 / 7 / 8 /{ }^{2,3,4}$ and of the programmatic panel discussion in Santa Fe $1987^{5}$.

The most important (objective) demand to a solution $f^{M}(g)$ is its positivity $f^{M}(g) \geq 0$. In the next we everywhere suppose that this demand is obeyed by the solutions discussed. We also suppose that the information from pole figure zeros is used in a maximum way. In this case the positivity demand works especially constructive leading 
to a direct coupling between $\hat{\tilde{f}}$ and $\tilde{f}$ in the zero region $G_{0}$ of the orientation space ${ }^{6,7}$. $G_{0}$ contains all points $g_{0}$ connected with the pole figure zeros at $\mathbf{y}_{0}=g_{0}^{-1} \mathbf{h}_{i}$ :

$$
\widetilde{P}_{\mathbf{h}_{i}}\left(\mathbf{y}_{0}\right)=0, f\left(g_{0}\right)=0, \widetilde{\widetilde{f}}\left(g_{0}\right)=-\tilde{f}\left(g_{0}\right), g_{0} \in G_{0} .
$$

In case of a great zero region (many pole figure zeros, sharp textures) the variation width of $f^{M}(g)$ really disappears. I.e. all mathematically proper reproduction or ghost correction methods should provide practically identical (positive) solutions. Differences come from method specific approximations or concern the computing efficiency of the corresponding algorithms.

Therefore only the case of weak textures without pole figure zeros, i.e. $\left(0<\min \left\{\widetilde{P}_{\mathbf{h}}(\mathbf{y})\right\} \equiv P \leq 1\right)$, and with a great variation width remains problematic. Such a width can mathematically be narrowed in the most elegant way by extremum conditions. But those should desirably follow from some physical arguments.

\section{STRUCTURE AND CORRELATION ELEMENTS OF ORIENTATION DISTRIBUTIONS}

The isotropic background ("phon") $0 \leq F \equiv \min \{f(g)\} \leq P \leq 1$ and regions with enlarged intensity around certain maxima ("texture components") are the typical elements of orientation distributions. The extremum cases are the random distribution ("no texture") $f(g)=1$ or an effective single crystal $f(g)=8 \pi^{2} \delta\left(g, g_{0}\right)$. Statistical fluctuations and faded characteristics of processes lead to texture components with finite widths ("short range correlations"). Crystal symmetry, symmetries of external fields or of processes modifying texture (e.g. twinning) may lead to certain "long range" correlations between intensities in the G-space. Such correlations (from the view of a starting crystallite or of a starting sample coordinate system) can be detected by the simplest crystal or sample sided correlation functions $(\kappa \in G)$ :

$$
K(\kappa)=\frac{1}{8 \pi^{2}} \int_{G} f(\kappa \cdot g) f(g) d g, \bar{K}(\kappa)=\frac{1}{8 \pi^{2}} \int_{G} f(g \cdot \kappa) f(g) d g .
$$

For the next it will be of importance that correlations in real ODFs are only of "finite dimension". I.e. the functional form of the orientation distribution in the vicinity of $g$ is not (point by point !) coupled with the orientation distribution in the whole remaining part of the G-space. In this case crystallites possessing orientations belonging to a texture component (together with its symmetrically equivalent ones) or to a fibre component realize 1 : finite or $1: \infty$ correlations respectively. A strong coupling between all ODFvalues in the G-space will correspond to an $\infty: \infty$ correlation of "infinite dimension" and seems not to be realistic from a physical view.

Any real ODF is the result of certain processes modifying a starting distribution that in principle consists of independent elements. One type of texture modifying processes transforms the starting distribution by strong geometric relations (e.g. twinning or Kurdjumow Sachs relation). At this the independent character of the starting distribution will practically be preserved. For another type of processes (e.g. rolling) the texture modification takes places by a great number of consecutive steps. These steps can be understood as the result of a driving field in the orientation space (cf. the flow field in Taylor theory ${ }^{8}$ ). This field may possess clear inner couplings and may e.g. satisfy a differential equation. During the texture transformation this field will of course stamp its properties on the starting distribution. But at the beginning the independence of the starting distribution prevails and in the final stage only peaks with finite widths at stable orientations will be seen in the ODF as the only remaining information about the 
specific properties of the given transformation field. I.e. again we will find correlations of finite dimension only.

On the other hand it is known that peak-like components are falsified in a specific manner in reduced ODFs $\widetilde{f}(g)$. These distortions can be interpreted as $\infty: \infty$ correlations. I.e. the physical realization of them will be exceedingly improbable. Therefore it seems that conditions for ghost correction minimizing such correlations will physically be more founded than conditions of pure subjective character.

\section{SPECIFIC CORRELATION IN REDUCED ORIENTATION DISTRIBU- TION FUNCTIONS}

One of the most nice results in connection with the analysis of the central problem of texture analysis (1) was the fact that it succeeded to express $\widetilde{f}(g)$ explicitly by $f(g)$ :

$$
\begin{gathered}
\tilde{f}(g)=f(g) / 2+f_{G}(g), f_{G}(g)=f_{\pi}(g)+f_{\Omega}(g), \\
f_{\pi}(g)=\int_{N} f([\omega=\pi, \mathbf{n}] \cdot g) d \mathbf{n} /(8 \pi) \geq 0, \\
f_{\Omega}(g)=\int_{0}^{\pi} d \omega \int_{N} d \mathbf{n} t^{2} \frac{\omega}{2}\{f([\omega=\pi, \mathbf{n}] \cdot g)-f([\omega, \mathbf{n}] \cdot g)\} /\left(8 \pi^{2}\right),
\end{gathered}
$$

with $[\omega, \mathbf{n}]-\mathbf{a}$ rotation about an axis $\mathbf{n}$ trough the angle $\omega$. Although this connection was published and discussed more than ten years ago ${ }^{9-12}$ the "correlation aspect" of this result ${ }^{1}$ is less known.

Indeed, speaking about $\widetilde{f}(g)$ commonly we are only thinking in terms of the "l-even part" of the series representation of $f(g)$ :

$$
f(g)=\sum_{l=0}^{\infty} \sum_{\mu, \nu} C_{l}^{\mu, \nu} D_{\mu, \nu}^{l}\left(g^{-1}\right), \tilde{f}(g)=\sum_{l=\alpha(2)}^{\infty} \sum_{\mu, \nu} C_{l}^{\mu, \nu} D_{\mu, \nu}^{l}\left(g^{-1}\right),
$$

that may be negative also. Equ. (5) shows that $\tilde{f}(g)$ contains one half of the true ODF plus an intensity coming from points in the G-space preferably with orientation distances $\omega \approx \pi$. If we compensate negative values in a model $\hat{f}(g)$ adding a correspondingly positive background we get after renormalization a nonnegative $f^{M}(g) \geq 0$ in the whole G-space. I.e. theoretically this distribution could represent a real ODF. But can such a distribution belong to a real sample?

Figure 1 once more shows the "SANTA FE" hard test distribution ${ }^{4}$ with record ghost effects and the specific property $\widetilde{f}(g) \geq 0$. Adding in Figure $1 \mathrm{~b}$ some more isolines between 0.1 and 0.5 optically the "disorder" would be much greater yet. The ODF in Figure 1a consists of a phon and one simple texture component at $g_{0}=\{122\}<221>$ with a halfwidth of $20^{\circ}$ (short range correlations) multiplied by (cubic) crystal and (orthorhombic) sample symmetry. The reduced ODF in Fig. 1b not only shows a much more complicated distribution but the critical circumstance is that all (!) functional values of $\widetilde{f}(g)$ are coupled among one another. That is just we have characterized by the term $\infty: \infty$ correlations.

The analytical expression for this fact follows from (5) if we always set $\widetilde{f}(g)$ for $f(g)$ :

$$
\tilde{f}(g)=2 \tilde{f}_{G}(g) .
$$

For any $g$ in the left the right part couples all values of $\tilde{f}(g)$ in the G-space. An analogic expression does not exist for a true ODF because all $f(g)$ values are in principle independent ones. A second reason for an "unphysical" behaviour of $\widetilde{f}(g)$ for peak-like 
a)

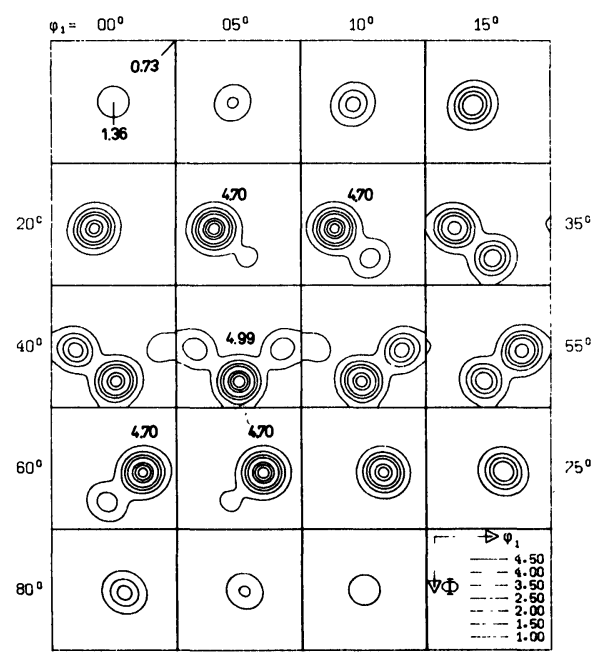
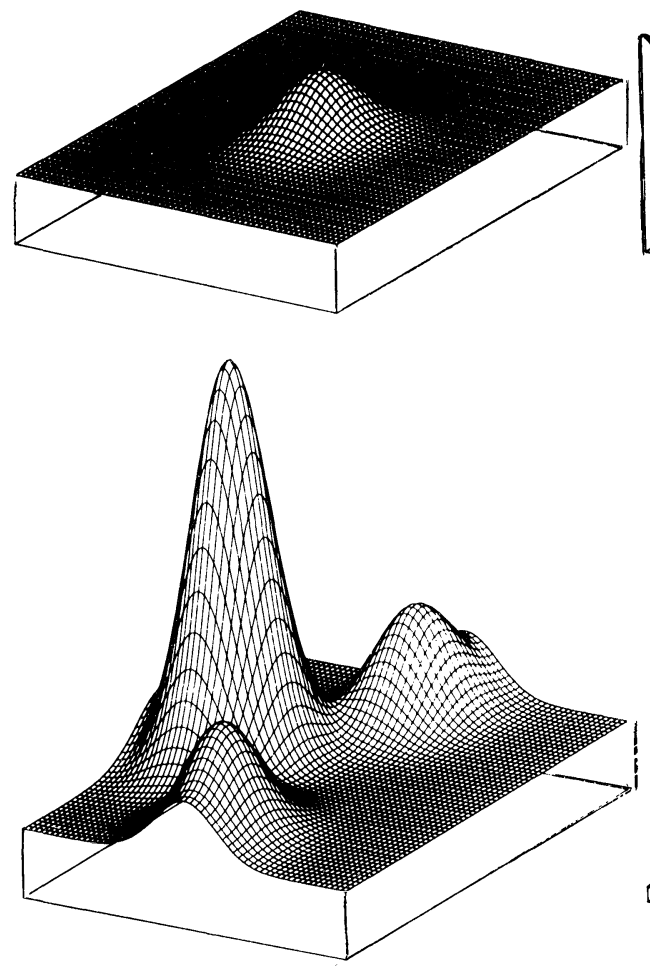

b)
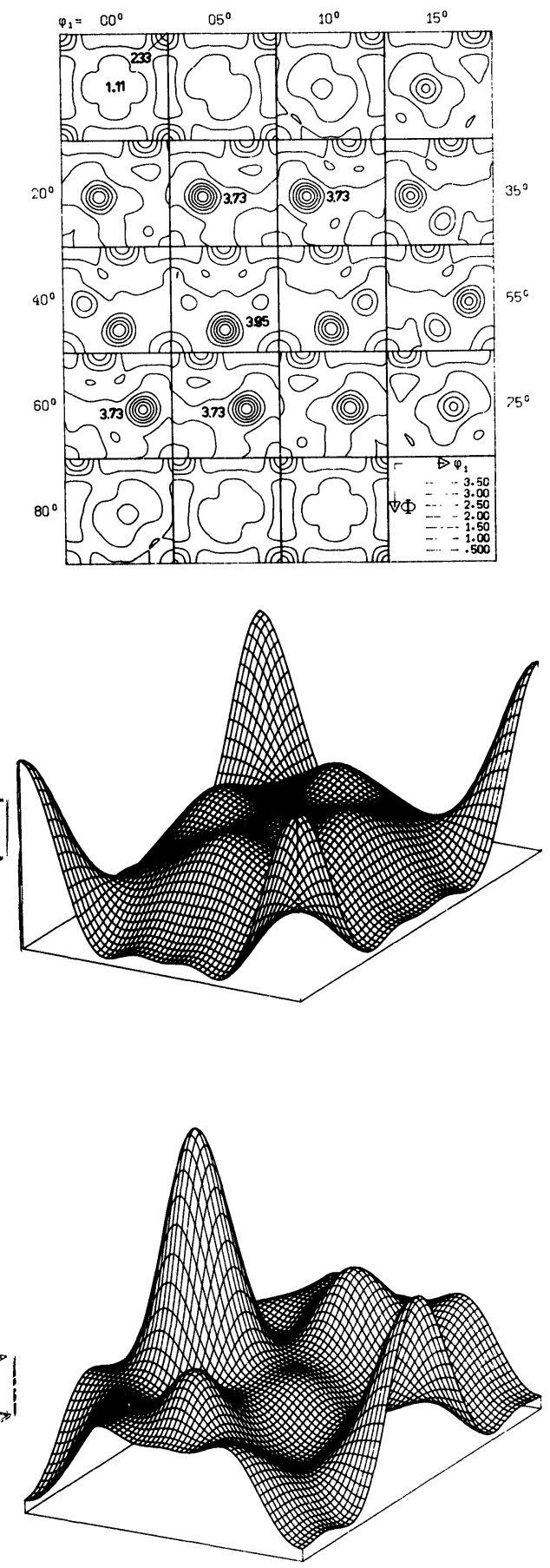

Figure 1. $f(g)$ a) and $\tilde{f}(g)$ b) of the SANTA FE test distribution. Separately the $\varphi_{2}=0^{\circ}, 45^{\circ}$ sections are given. 
distribution structures follows from the properties of $f_{\Omega}(g)$ at $\omega \approx \pi$. The $t g$ factor in (7) effectively represents the result of a folding operation about a derivation of $f(g)$ at $\omega \approx \pi$. This refers to more complicated correlations than simple short range ones only resulting from statistical fluctuations in an ensemble of independent members.

With the help of standard functions ${ }^{1}$ all these moments can systematically be analysed. Moreover, qualitatively they can be demonstated by one-dimensional relations and figures if only the dependence from the orientation distance $\omega$ is considered.

Figure 2 shows the typical properties of the ghost part $f_{G}(\omega)$ for a peak-like distribution $f(\omega)$ with the maximum at $\omega=0$. The oscillation at $\omega=\pi$ is characteristic for the "unphysical" behaviour mentioned above.

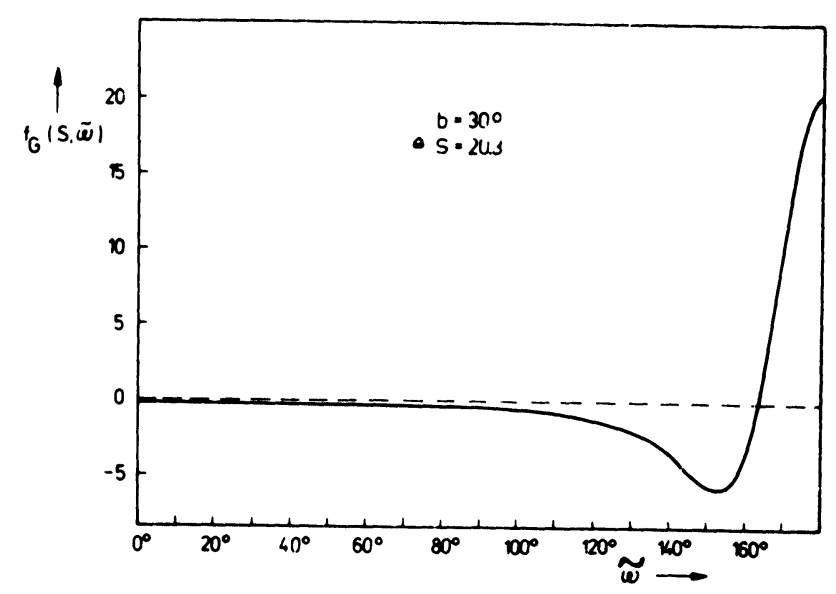

Figure 2. The ghost part $f_{G}(\omega)$ of the reduced ODF for a Gaussian standard distribution ${ }^{1}$.

Because it will be typical for all peaks of an ODF, correlation functions like (4) realizing a mean over all peak positions should also show such oscillations for reduced ODFs. I.e. the analysis of the simplified correlation function $\mathrm{K}$ for $f^{M}(g)$

$$
\begin{gathered}
K_{\omega}=\widetilde{K}_{\omega} \equiv \int_{N} K(\kappa) d \mathbf{n} /(4 \pi)=\sum_{l=0} K^{l l} \chi_{l}(\omega), \\
\chi_{l}(\omega)=\sin ((2 l+1) \omega / 2) / \sin (\omega / 2), \kappa=[\omega, \mathbf{n}] \\
K^{l}=\sum_{\mu, \nu}\left|C_{l}^{\mu, \nu}\right|^{2} /(2 l+1)^{2}
\end{gathered}
$$

especially at $\omega \approx \pi$ may help to detect unphysical $\infty: \infty$ correlations. Moreover, mathematical criteria of minimizing such effects may be used as conditions for ghost corrections.

\section{CORRELATION RELATED CONDITIONS}

The simplest variant of a peak oriented condition is

$$
K_{\pi}=\widetilde{K}_{\pi}+\widetilde{\widetilde{K}}_{\pi} \equiv \sum_{l=\alpha(2)}^{\infty} K^{l}-\sum_{l=1(2)}^{\infty} K^{l}->\min ,
$$


with $K_{\omega} \geq 0$, due to $f^{M}(g) \geq 0$. Because of $K^{-l} \geq 0$ (13) tries to reconstruct a $C_{l}^{\mu, \nu}$ spectrum (l odd) near to the $l$ even spectrum. Test calculations have shown that "true" $\omega=\pi$-correlations connected with $C_{2}$-axes in crystal or sample symmetry do not disturb the condition (13). However, as will be seen from Table 1 the single criterion (13) does not possess a distinct minimum structure within the variation width. Another formulation can be found directly minimizing the "unphysical" correlations connected with relations of type (9)

$$
\widetilde{f}(g)=2 \widetilde{f_{G}}(g) ; \widetilde{\widetilde{f}}(g)=-2 \widetilde{\widetilde{f}}(g)
$$

that should not be characteristic for "true" ODFs. I.e. the covariance of $f^{M}(g)$ and $f_{G}^{M}(g)$ should be a minimum:

$$
\left|\operatorname{cov}\left(f^{M}, f_{G}^{M}\right)\right| \equiv\left|E\left(f^{M}(g) \cdot f_{G}^{M}(g)\right)-E\left(f^{M}(g)\right) \cdot E\left(f_{G}^{M}(g)\right)\right|->\min ,
$$

with

$$
E(\varphi(g)) \equiv \int_{G} \varphi(g) d g /\left(8 \pi^{2}\right)
$$

By the expression for the texture index it follows

$$
\begin{gathered}
f_{2}=E\left(f^{2}(g)\right)=K_{\omega=0}=\sum_{l=0}^{\omega}(2 l+1) K^{l}=\tilde{f}_{2}+\underset{f_{2}}{\approx}, \\
\left|\operatorname{cov}\left(f^{M}, f_{G}^{M}\right)\right|=\left|\tilde{f}_{2}-\tilde{f}_{2}^{M}-1\right| / 2->\min .
\end{gathered}
$$

For random pole figures $\left(\widetilde{P}_{\mathbf{h}}(\mathbf{y})=1, \tilde{f}_{2}=1\right)(18)$ immediately leads to the random distribution $\}_{2}^{M}=0, f^{M}(g)=1$. I.e. the exotic "Tokyo" distributions (cf. Fig. $5^{3}$, Fig. 48-50 in ${ }^{10}$ ) all providing $\widetilde{P}_{h}(y) \equiv 1$ and possessing a high degree of $\infty: \infty$ correlations will automatically be excluded.

In the common case (peak structures and phon) the condition (18) must be weighted by the factor characterizing the "truly texturized" part of the crystallites:

$$
C O V \equiv\left(1-f_{\min }^{M}\right)\left|\operatorname{cov}\left(f^{M}, f_{G}^{M}\right)\right|->\min .
$$

The tendency $\tilde{\tilde{f}_{2}}->\widetilde{f}_{2}$ can qualitatively be formulated as $f_{2}->\max$ also. From such a view the condition (19) aspires to a "sharp texture (minimum number of peaks, but these with great intensity) with a maximum phon". I.e. it just means the intuitive basis conditions for the WIMV method ${ }^{13,14,4}$ as well as the component fit concepts ${ }^{15}$.

In order to demonstrate the variation width of solutions model distributions can be used constructed by the known parts of a given ODF $(\widetilde{f}(g), \widetilde{f}(g))$

$$
f^{M}(c, g)=\tilde{f}(g)+c \tilde{\widetilde{f}}(g)=c f(g)+(1-c) \tilde{f}(g) ; \text { climited by } f^{M}(c, g) \geq 0 .
$$

Table 1 shows the minimum structure of (19) for the SANTA FE distribution (Fig.1). The "true" ODF (physically most probable) corresponds to $c=1$.

Of course the condition (19) loses its sense for $f(g) \geq 0$ and if $f_{G}(g)$ (cf.(9)) reduces to realizable correlations of finite dimension like for fibre components (imitation of double fir tree components by $\widetilde{f}(g)^{16,17,1}$ ). 
Table 1. Characteristic quantities of the SANTA FE model distribution within the variation width. $f^{M}(c, g)=c f(g)+(1-c) \widetilde{f}(g)$

\begin{tabular}{ccccccccc}
\hline & & & & & & & & \\
$c$ & $f_{\text {min }}^{M}$ & $f_{\text {max }}^{M}$ & $f_{2}^{M}$ & entr. & cube & $K_{\pi}$ & COV & $f^{M}(c, g)$ \\
\hline 0.0 & 0.10 & 3.95 & 1.251 & -0.114 & 2.33 & 1.0195 & 0.225 & $f(g)$ \\
0.2 & 0.23 & 4.16 & 1.255 & -0.107 & 2.01 & 1.0193 & 0.190 & \\
0.4 & 0.35 & 4.37 & 1.265 & $-\mathbf{0 . 1 0 4}$ & 1.69 & 1.0187 & 0.153 & \\
0.6 & 0.48 & 4.58 & 1.282 & -0.106 & 1.37 & 1.0178 & 0.144 & \\
0.8 & 0.61 & 4.78 & 1.307 & -0.110 & 1.05 & 1.0165 & 0.077 & \\
1.0 & $\mathbf{0 . 7 3}$ & 4.99 & 1.337 & -0.119 & 0.73 & 1.0150 & $\mathbf{0 . 0 4 4}$ & $f(g)$ \\
1.1 & 0.57 & 5.10 & 1.355 & -0.124 & 0.57 & 1.0140 & 0.062 & \\
1.3 & 0.26 & 5.30 & 1.397 & -0.138 & 0.26 & 1.0114 & 0.078 & \\
1.5 & $<0$ & & & & & & & \\
\hline
\end{tabular}

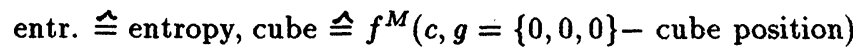

\section{CONDITIONS IN REALIZED CORRECTION CONCEPTS}

A sufficient analysis of existing ghost correction concepts was already given on ICOTOM $7 / 8^{3,4}$, cf. ${ }^{5,18,19}$ also. There is not necessity in detail to repeat the given statements about earlier developed correction concepts. But it is worth to remember two general moments.

- Due to the ambiguity of our problem any algorithm satisfies additional conditions just permitting its "unique" solution that will reproducible be got by a computer. The conditions may be explicitly formulated like in the WIMV method or may be of "hidden" character like in the conventional vector method ${ }^{18}$. A necessary demand to all methods with hidden conditions is that the characteristic properties of the corresponding solutions should be known. With simple words, will be the method specific solution for the SANTA FE test example near to fig. 1a or to $1 \mathrm{~b}$ ?

- The analysis of the nature of the hidden conditions will be less complicated remembering that (already due to the positivity demand $f^{M}(g) \geq 0$ ) any ghost correction concept leads to a nonlinear problem. Its numerical solution will in any case be determined in an iterative manner. Now for cases with a great variation width the resulting $f^{M}(g)$ will decisively depend on method specific starting approximation and on the structure and properties of the corresponding correction operator.

Two new valuable correction concepts were first introduced on ICOTOM $8^{20,21}$ and developed in the last years ${ }^{22-24}$. The original variant of the iterative positivity method of Bunge and Dahms is of the "hidden" type. It uses the reduced ODF as zero approximation. The correction procedure does not contain distinct specific inner symmetries. Therefore it will provide like the $h^{2}$-method of Van Houtte ${ }^{25}$ and his new $f(g)=\exp (y(g))$-concept ${ }^{26}$ a positive solution near to $\widetilde{f}(g)$. In critical situations with $\tilde{f}(g) \geq 0$ (cf. Fig. 1) there is not necessity and not point of contact to start the ghost correction (cf. in this connection the contribution of Wagner, Humbert, Esling and Muller).

The maximum entropy concept is of somewhat mysterious character and needs a more detailed analysis. 


\section{MAXIMUM ENTROPY AND GHOST CORRECTION}

The maximum entropy concept succesfully works in information theory and image reconstruction ${ }^{27}$. A linear equation system for $\mathrm{N}$ unknowns $x_{n} \geq 0$ :

$$
y_{m}=\sum_{n=1}^{N} a_{m n} x_{n} ; m=1,2, \cdots, M<N
$$

will be completed by an additional extremum condition in order to get an unique solution:

$$
S=-\sum_{n=1}^{N} x_{n} \ln x_{n}->\max .
$$

The solution can be represented in an (positive !) exponential form

$$
x_{n}=\exp \left\{\lambda_{1}, \lambda_{2}, \cdots, \lambda_{M}, a_{m n}\right\} \geq 0
$$

and the $\lambda_{m}$ must be determined by the system of nonlinear equations following from (21), (23). S can be interpreted as the degree of "disorder or ignorance" and the maximum condition prefers solutions with a "soft" (not sharp) $x_{n}$ spectrum.

Wang, $\mathrm{Xu}$ and Liang ${ }^{23}$ first used the analogy of (21) with the central problem (1). Introducing cells $(n)$ in the G- respectively in the pole figure space $(m)$ the $a_{m n}$ will be the "elementary pole figures" well known from the vector method ${ }^{18}$.The poblem can be transformed into the Fourier space also $\left(y_{m} \widehat{=} C_{l}^{\mu, \nu}\right.$ for $l$ - even, the $a_{m, n}$ are quantities related to spherical funtions) leading to a ghost correction method. Cf. the contribution of Wang Fu et al. on our conference.

The analysis of the $S_{\max }$ condition in the sense of our previous considerations is somewhat complex and can be given in form of statements only.

A great entropy means as a qualitative tendency a small texture sharpness $f_{2}$. I.e. due to (17) $\widetilde{f}_{2}^{M}$ will be lowered. This leads to a solution near by $\widetilde{f}(g)$ and consequently a smoothing operation does not automatically mean a suppression of ghost peaks $(\infty: \infty$ correlations). The specific form of the loss of information in texture analysis (all l - odd informations in pole figures) leads to certain inner symmetries (9). Such a systematic loss cannot absolutely be repaired by a "stochastic" smoothening like in the above mentioned fields. However, the equivalence $S->\max \widehat{=} f_{2}->\min$ concerns great $f(g)$ values only. Small values prefer a lift. I.e. the entropy will reach its maximum for a solution $f^{M}(g)$ within the variation width at some distance from $\widetilde{f}^{\widetilde{f}}(g)\left(f_{2}^{M}->\min \right)$ and $f(g)$ (min of $\infty: \infty$ correlations). Table 1 verifies this conclusion examined for a great number of other test examples also. Figure 3 shows the $S_{\max }$ solution for the SANTA FE example with remarkable remaining ghosts in the cube position.

For examples with $\widetilde{f}(g)><0$ the variation width of solutions will be relatively small and any positive solution will automatically be near to the true ODF. At this the $S_{\text {max }}$ condition is of secondary importance but leads to an elegant and compact algorithm (23).

Practically simultaneously with the chinese group Schaeben ${ }^{24}$ considers the $S_{\max }$ concept in the direct formulation with cell intensities in the G-space and pole figures. The corresponding solution will be determined iteratively :

$$
\begin{aligned}
x_{n}^{(k+1)} & =\prod_{m=1}^{M}\left(y_{m}^{e x p} / y_{m}^{(k)}\right)^{r_{k} \cdot a_{m n}} x_{n}^{(k)}, 0<r_{k} \leq 1, \\
y_{m}^{(k)} & =\sum_{n=1}^{N} a_{m n} x_{n}^{(k)}, x_{n}^{(0)}=\prod_{m=1}^{M}\left(y_{m}^{e x p}\right)^{r_{0} \cdot a_{m n}} .
\end{aligned}
$$



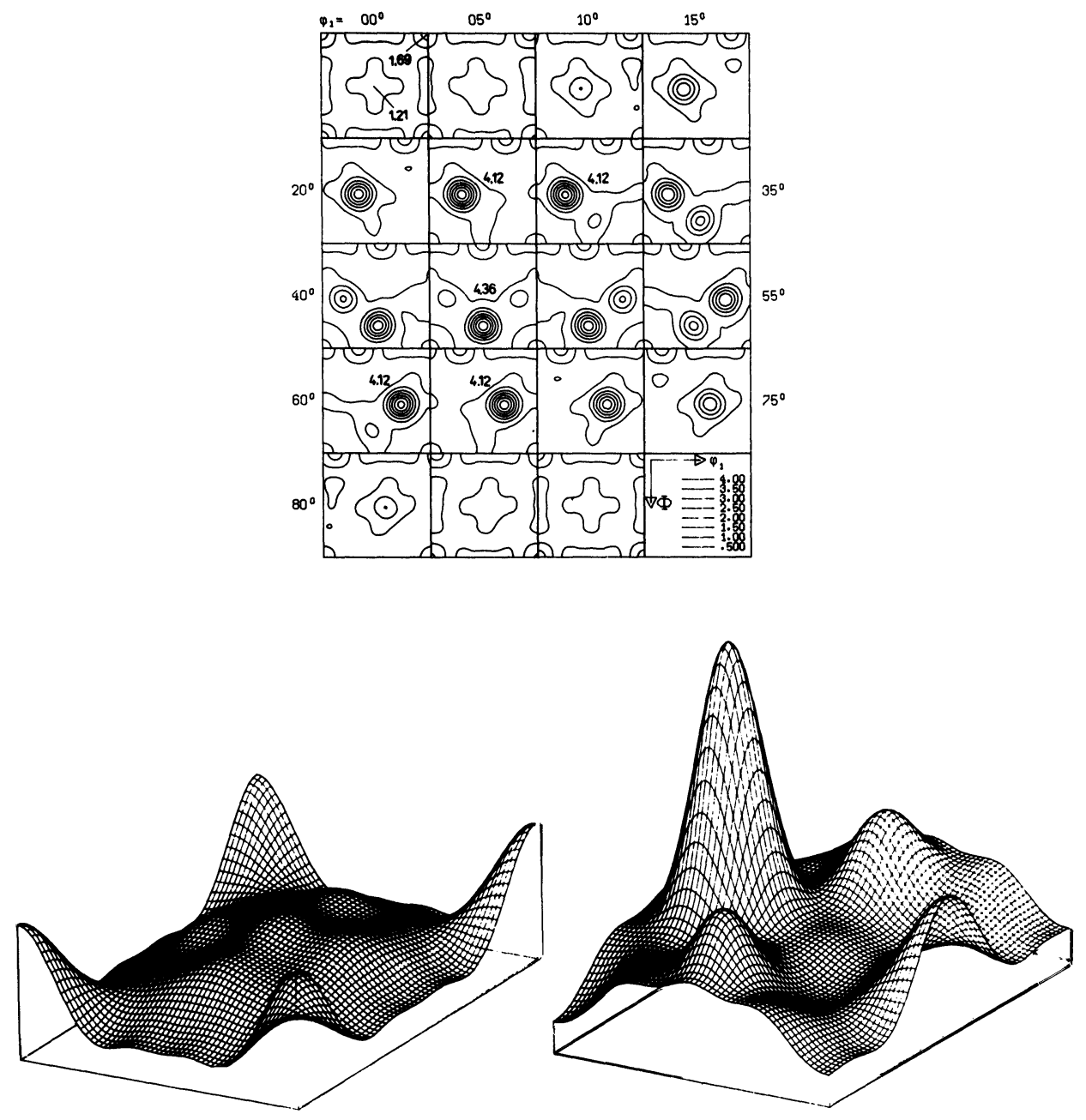

Figure 3. The ODF $f^{M}(c, g)$ of the SANTA FE model distribution for $\mathrm{c}=0.4$ (cf.(20) and Table1) with maximum entropy. Like in Fig. $1 \varphi_{2}=0 ; 45^{\circ}$-sections are separately given. 
Lent ${ }^{28}$ proved for a similar algorithm the convergence to the $S_{\max }$ solution. There is practically not difference to equivalent expressions in the WIMV method ${ }^{3}$. But (24),(25) leads to $S_{\max }\left(\hat{=} f_{2} \approx \min\right.$ ) whereas WIMV aspires to a $f_{2}->\max$ solution? The contradiction disappears remembering that WIMV works with $r_{k}>1$, i.e. with a sharp zero approximation also, which decisively determines the character of the solution. The $r_{k}(k>0)$ mainly influence the velocity of convergence only. From this point of view analogic algorithms like the ADC method ${ }^{29}$, (cf. the contribution of Pawlik, Pospiech, Luecke also) are to be analysed.

In Table 2 the two regimes $\left(S_{\max }\right.$ or $\left.W I M V\right)$ for (24),(25) are compared for the SANTA FE example using the four standard pole figures (111),(100),(110) and (311). Cf. the corresponding characteristics of the solutions with table 1 also. The role of the zero approximation is obvious. The WIMV solution ( $\mathrm{cf.}^{4}$ ) is practically identical with Fig. 1a, the $S_{\max }$ result is near to Fig. 3 .

Table 2. Comparison of the iteration path for the maximum entropy or the typical WIMV regime. SANTA FE distribution, 4 pole figures, $5^{\circ}-$ grid,$P=0.73$.

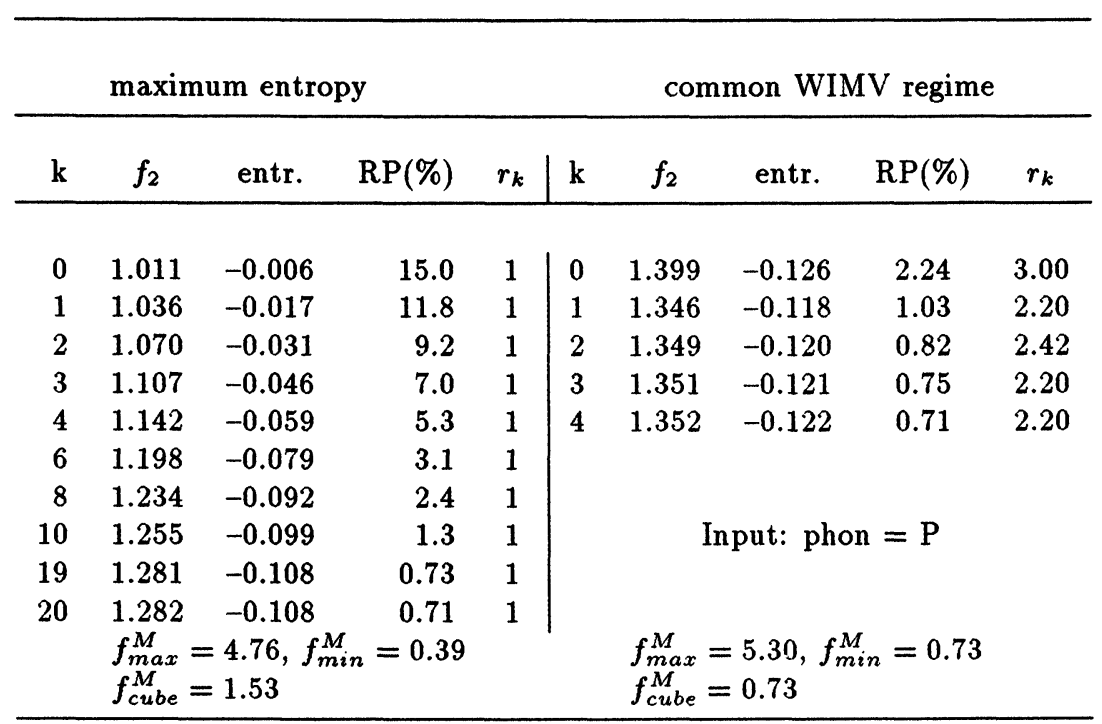

Remark:

$S$ in (22) corresponds to a definition using G-space cells of equal volume $v_{n}=8 \pi^{2} / N\left(x_{n}=f_{n} \cdot v_{n} /\left(8 \pi^{2}\right)\right)$. Working with arbitrary cells (e.g. $5^{\circ}$-grid) $\mathrm{S}$ is to be defined by

$$
S=-\int_{G} f(g) \ln f(g) d g /\left(8 \pi^{2}\right)=-\sum_{n=1}^{N}\left(f_{n} v_{n} \ln f_{n}\right) /\left(8 \pi^{2}\right)
$$

and will be a negative quantity in this case. 


\section{CONCLUSION}

Conditions (additional to $f^{M}(g) \geq 0$ ) for ghost correction are of especial importance for medium and weak $(\widetilde{f}(g) \geq 0)$ textures with a great variation width of solutions. They should desirably rest upon physical arguments. Such a condition seems to be the suppression of $\infty: \infty$ correlations. For peak oriented concepts this is equivalent to the demand of a high phon and texture index. The formal condition of maximum entropy somewhat differs from these characteristics and may lead to remaining ghosts.

The phon iteration procedure ${ }^{3}$ of the WIMV method (considering renormalized reproduction problems with always decreasing phon in the remaining pole figures) effectively leads to the case of sharp textures with a neglecting variation width. This concept can also be realized without problems by all other methods providing positive solutions $f^{M}(g) \geq 0$.

At the end it seems to be worth to refer to some danger connected with an overevaluation of the "power" of mathematical tools. Additional conditions (explicit or hidden ones) commonly possess an extremum character. I.e. problems of type (1),(21) will then be mathematically well determined. Therefore an "ODF" can always be found independently on the quantity (and quality) of the experimental input data in comparison to the number of unknowns that will be determined by the "intelligent" computer.However, we should not forget the rule of thumb that the number of experimental data must at least be in the order of the number of unknowns. If this ratio is destroyed the results are to be considered with sound scepticism. Only pole figure zeros drastically reduce the effective number of unknowns.

In any case it is to be recommended to examine the quality of solutions for the given method and class of data sets (symmetry, grid, number and sort of pole figures, degree of their completeness, overlapping,sharpness of texture a.s.o.) by hard test examples.

We also recommend in more detail to analyse the properties of "true" ODFs derived by single orientation measurements or e.g. Taylor calculations in order to formulate physically founded conditions for ghost correction. The author hopes that the consideration of correlations will be an interesting field in this direction.

\section{REFERENCES}

1. S.Matthies, G.W.Vinel and K.Helming, Standard Distributions in Texture Analysis, Vol. 1 (1987), Vol. 2 (1988), Vol. 3 (1990),(Akademie Verlag, Berlin)

2. S.Matthies, Proc. of the 6th ICOTOM (Tokyo, Iron and Steel Institute of Japan, 1981), p. 276.

3. S.Matthies, Proc. of the 7th ICOTOM (Noordwijkerhout, Netherland Soc. for Materials Science, 1984), p. 737.

4. S.Matthies, Proc. of the 8 th ICOTOM (Santa Fe, The Metallurgical Society, Inc., Warrendale, 1988), p. 37.

5. H.R.Wenk, H.J.Bunge, J.S.Kallend, K.Luecke, S.Matthies, J.Pospiech, and P.Van Houtte, p. 17 in ref. 4.

6. H.J.Bunge and C.Esling, J. Physique Lettres 40,627 (1979)

7. H.J.Bunge, Texture Analysis in Materials Science (Butterwoths, London, 1982) 
8. H.Klein, C.Esling and H.J.Bunge, p. 307 in ref. 4.

9. S.Matthies, phys. stat. sol (b), 92, K135 (1979)

10. S.Matthies, Aktuelle Probleme der quantitativen Texturanalyse (ZfK-480, Rossendorf, 1982)

11. C.Esling, J.Mueller and H.J.Bunge, J. Physique 43, 189 (1982)

12. J.Jura, K.Luecke and J.Pospiech, Z. Metallkde. 71, 714 (1980)

13. S.Matthies and G.W.Vinel, phys. stat. sol. (b) 112, K111 (1982)

14. H.R.Wenk ed., Preferred Orientation in Deformed Metals and Rocks (Academic Press, San Diego, 1985)

15. K.Luecke, J.Pospiech, J.Jura and J.Hirsch, Z. Metallkde77, 312 (1986)

16. S.Matthies, phys. stat. sol. (b)98, K113 (1980)

17. S.Matthies, A.Muecklich and K.Hennig, Z. Metallkde. 74, 803 (1983)

18. H.Schaeben, phys. stat. sol. (b) 123, 425 (1984)

19. S.Matthies, H.R.Wenk and G.W.Vinel, J. Appl. Cryst. 21, 285 (1988)

20. H.R.Bunge, p. 69 , in ref. 4.

21. F.Wang, J,Xu and Z.Liang, p. 111 in ref. 4.

22. M.Dahms and H.J.Bunge, p. 79 in ref. 4.

23. F.Wang, J.Xu and Z.Liang, Textures and Microstructures 10, 217 (1989)

24. H.Schaeben, phys. stat. sol. (b) 148, 63 (1988)

25. P.Van Houtte, Textures and Microstructures 6, 1 (1988)

26. P.Van Houtte, private communication (1990)

27. G.Minerbo, Comput. Graph. Image Proces. 10, 48 (1979)

28. A.Lent, SPSE conf. Proc. Image Analysis and Evaluation (Soc. Photog. Sci. Engng., Washington (D.C.) 1977), p. 249.

29. K.Pawlik, phys. stat. sol. (b) 134, 477 (1986) 